\title{
Enteroparasitosis en niños bajo 5 años de edad, indígenas y no indígenas, de comunidades rurales del Paraguay
}

\author{
Gloria Echagüe, Liliana Sosa, Valentina Díaz, Irene Ruiz, Lourdes Rivas, Dominich Granado, \\ Patricia Funes, Jorge Zenteno, Norma Pistilli y Mirtha Ramírez
}

\section{Enteric parasitic disease in children under 5 years of age, indigenous and non-indigenous, from rural communities in Paraguay}

Background: Official figures of mortality in children under five years of age in the Americas, report that infectious and parasitic diseases caused most of the deaths. Objective: To evaluate the frequency of intestinal parasites in vulnerable children, indigenous and non-indigenous, and their socio-environmental characteristics. Patients and Methods: We evaluated 247 children under five years of age, of both sexes. Descriptive study with an analytical component, transverse cutting. Copro-parasitological examinations were carried out and semi-structured interviews to collect socio-demographic data were conducted. Results: The frequency of intestinal parasitic diseases was $56.1 \%$ and $35.5 \%$ in indigenous and non-indigenous children, respectively. In both populations, the most common pathogens were Blastocystis hominis and Giardia lamblia. Conclusion: We found a high frequency of parasitism in indigenous children at the expense of protozoa. Non-indigenous children still present the same parasitic species found in previous studies, suggesting the need to implement more control and prevention. The poor conditions in which they live favor the development of these diseases.

Key words: Parasitic disease, vulnerable populations, helminths.

Palabras clave: Parásitos, población vulnerable, protozoarios, helmintos, enteroparasitosis.

\section{Introducción}

$\mathrm{L}$ as infecciones por parásitos intestinales son endémicas alrededor del mundo y han sido descritas como parte de una de las mayores causas de morbi-mortalidad $^{1}$.

Afectan principalmente a la población infantil debido a su inmadurez inmunológica y poco desarrollo de hábitos higiénicos ${ }^{2}$. Los parásitos intestinales afectan desproporcionadamente a los más desfavorecidos, particularmente en las áreas rurales y los barrios pobres y marginalizados, y atrapan a las personas vulnerables en un ciclo de pobre$\mathrm{za}^{3}$. Por lo menos 46 millones de niños de las Américas corren el riesgo de padecer infecciones parasitarias ${ }^{3}$. Según la OMS, la prevalencia de las enteroparasitosis en América Latina oscila entre 20 y $30 \%$ de la población general y 60 a $80 \%$ para poblaciones con alta endemia ${ }^{4}$. En niños bajo cinco años de edad, cifras oficiales de mortalidad refieren que la mayor parte de las defunciones fueron causadas por enfermedades infecciosas incluyendo las parasitarias, que representaron $13 \%$ del total. Entre las enfermedades infecciosas las intestinales constituyeron el grupo más importante $(7 \%)^{4}$.

En nuestro país, el grupo de niños y niñas bajo cinco años presenta, entre las principales causas de morbilidad, a la parasitosis intestinal $(9 \%)^{5}$.

En los niños infectados, los parásitos intestinales causan anemia, deficiencia de vitamina A, retraso en el crecimiento, malnutrición, y trastornos del desarrollo físico y cognitivo ${ }^{3}$. La giardiosis, la criptosporidiosis y la amebiosis se encuentran entre las infecciones entéricas de mayor prevalencia alrededor del mundo. Debido a su importancia en países menos desarrollados, estas enfermedades han sido recientemente incluidas por la Organización Mundial de la Salud dentro del grupo de "enfermedades olvidadas o descuidadas", que impide el desarrollo y retrasa cambios socio-económicos importantes en países en desarrollo ${ }^{6}$.

Estudios internacionales revelan una alta prevalencia de infección parasitaria en niños; trabajos realizados en niños no indígenas encontraron en Venezuela una prevalencia que osciló entre 42,6 y 97,4\% ${ }^{7}$, en Cuba fue de $67,4 \% \%^{8}$, en Pakistán se encontró una prevalencia de 52,8\%, donde Giardia lamblia y Ascaris lumbricoides fueron los parásitos más comunes ${ }^{1}$. El trabajo realizado en niños indígenas de todas las edades en Colombia, reportó $100 \%$ de prevalencia de parásitos intestinales en cuatro comunidades ${ }^{9}$.

En el país, los hogares del sector rural se encuentran en condiciones relativas más adversas que los hogares urba-
Universidad Nacional de Asunción, Paraguay (GE, LS, VD $I R, L R, D G, P F, J Z, N P$ ). Instituto de Investigaciones en Ciencias de la Salud (IICS) Fundación Acción contra el Hambre (MR).

Los autores declaran que no poseen ningún conflicto de intereses en relación al trabajo enviado para publicación.

Estudio financiado por la Agencia Española de Cooperación Internacional para el Desarrollo (AECID).

Recibido: 13 de marzo de 2015 Aceptado: 21 de septiembre de 2015

Correspondencia a:

Gloria Ana María Echagüe de Méndez

gamechague@yahoo.com 
nos; los niños se ven afectados por la falta de suministro de agua potable y saneamiento, con dificultad en el acceso a las comunidades debido a caminos de tierra y puentes en mal estado, así como limitaciones en la atención médica, favoreciendo la desnutrición crónica, un mayor número de episodios de diarrea, parasitosis intestinales, mayor morbilidad y talla baja ${ }^{10-12}$. A ello se suma las disparidades existentes entre la salud de los pueblos indígenas y la de la población general, afectando a una gran proporción de indígenas la malnutrición y las enfermedades parasitarias y transmisibles ${ }^{4}$. Los indígenas están asentados predominantemente en áreas rurales $(91,5 \%)$ y cerca de $50,7 \%$ de la población indígena vive en la Región Oriental; 47\% de esta población está conformada por jóvenes bajo 18 años y $63 \%$ de los niños indígenas viven en la pobreza extrema ${ }^{13,14}$

A nivel nacional los trabajos publicados en niños no indígenas presentaron frecuencias de parasitosis entre 64 y 76\%; como el realizado por Pistilli y cols. ${ }^{15}$, Ramírez y cols. ${ }^{16}$ y Canese y cols. ${ }^{17}$; mientras que Araujo y cols. ${ }^{18}$ realizaron su estudio en niños indígenas en edad escolar de la comunidad de isla Saka, Caazapá, encontrando 44,6\%.

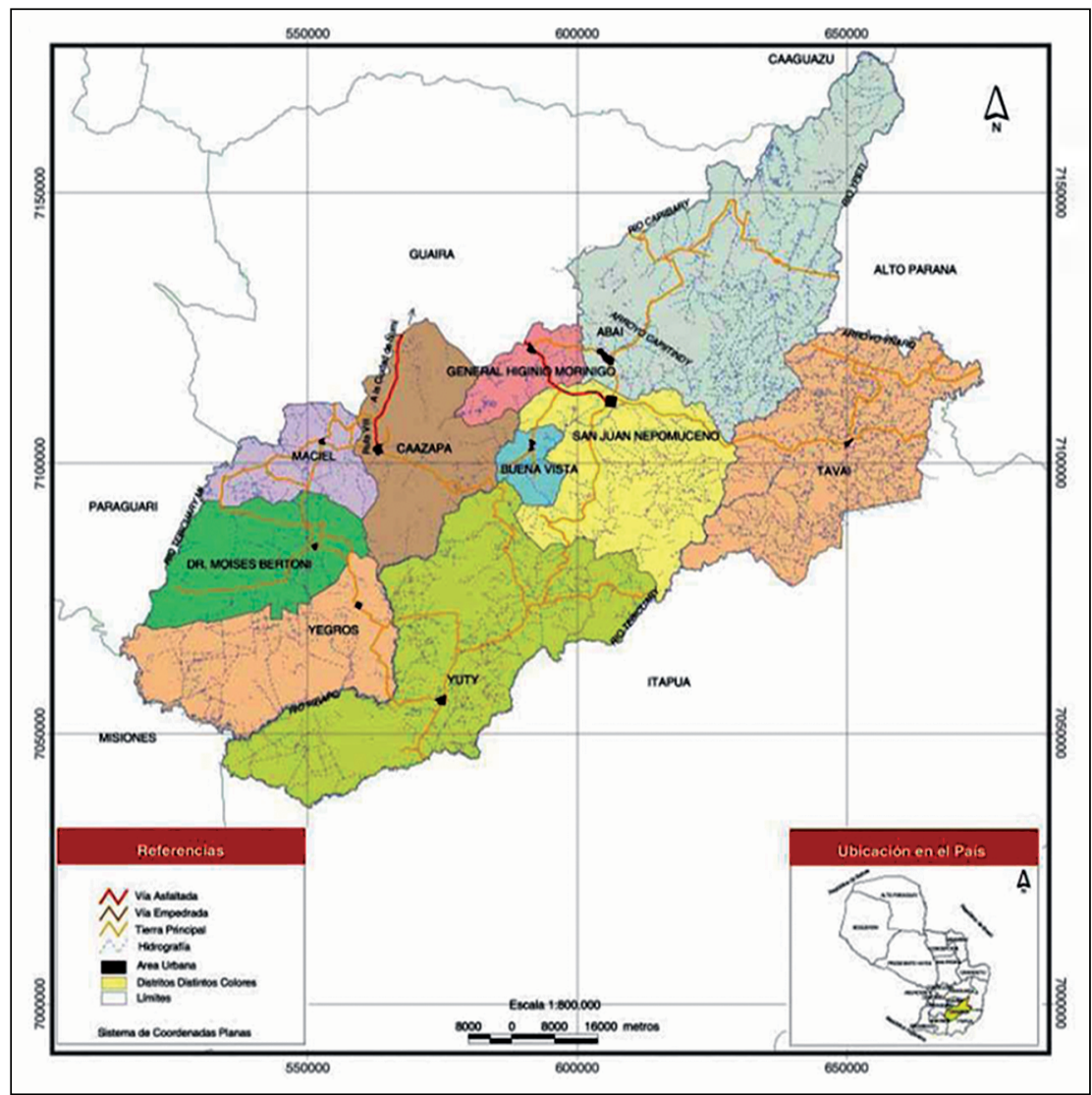

Figura 1. Mapa del Paraguay, Departamento de Caazapá (DGEEC).
Las infecciones parasitarias intestinales constituyen grandes y graves problemas médicos y de salud pública, en los países en desarrollo, particularmente en regiones tropicales como la nuestra ${ }^{19}$. Desde la familia, las niñas indígenas comienzan a tener limitaciones para su desarrollo integral, que se inicia en la infancia, en el hogar, donde se considera que las tareas domésticas son parte de ella y que la niña no necesita asistir a la escuela ${ }^{20}$. Las costumbres y cultura indígena constituyen un factor importante en el mantenimiento de los factores que favorecen las enfermedades.

Teniendo en cuenta la situación de la niñez en las zonas rurales y los factores condicionantes en las que viven estas poblaciones, este estudio tuvo como objetivo evaluar la frecuencia de enteroparasitosis en la población infantil vulnerable de comunidades rurales indígenas y no indígenas del Departamento de Caazapá y sus características socio-ambientales.

\section{Pacientes y Métodos}

\section{Tipo de estudio}

El diseño del estudio fue descriptivo con componente analítico, de corte transverso, con muestreo no probabilístico, de casos consecutivos, que consistió en la recolección de muestras de heces en los meses de marzo a mayo de 2012 y de 2013. El tamaño de la muestra fue estimado para un alfa bilateral de 0,05 , un nivel de confianza del $95 \%$ y un beta de 0,10 de acuerdo a la tabla 6 B de Hulley y col. ${ }^{21}$, para comparación de proporciones de variables dicotómicas.

\section{Área de estudio}

El trabajo se realizó en el Departamento de Caazapá, en las comunidades rurales de los distritos de Abaí y Tavaí. Caazapá constituye el sexto departamento de la República del Paraguay, se encuentra ubicado al sureste de la Región Oriental, a 228 km de la capital, Asunción, y está dividido en once distritos; cuenta con una población total de 139.517 habitantes, que representa $2,7 \%$ de la población total del país; solamente $17,9 \%$ de los pobladores del departamento habita en el área urbana, lo que evidencia el predominio de residentes en el área rural ${ }^{22}$. Todo el departamento está en la eco- región de la selva central, con un clima semi-tropical húmedo, de acuerdo a la Dirección General de Estadísticas, Encuestas y Censos del Paraguay (DGEEC) ${ }^{23}$ (Figura 1).

\section{Sujetos de estudio}

El universo estuvo representado por niños bajo cinco años de edad, pertenecientes a cuatro comunidades rurales indígenas y nueve comunidades rurales no indígenas cuyos padres o tutores accedieron a que participaran del 
estudio. De un total de 155 niños indígenas invitados participaron 123 niños, pertenecientes a la Familia Lingüística Guaraní, de la etnia Mbya Guaraní y Aché24, correspondientes a una comunidad del distrito de Tavaì: Tuna $i$, de la etnia Mbyá Guaraní y tres comunidades del Distrito de Aba i: Ypetimí de la etnia Aché, Ñu Apúa y Tajay de la etnia Mbyá Guaraní2 ${ }^{4}$. De los 133 niños no indígenas invitados, participaron 124 niños, correspondientes a tres comunidades del distrito de Tavaí: San Francisco, Santa Ana e Yvyhati y de siete comunidades del distrito de Abaí: Kilómetro 9, Michimí, San Francisco, San Marcos, San Miguel, San Miguel La Esperanza y Villa Pastoreo (Tabla 1).

\section{Estrategia de selección de muestra y diseño}

Se realizaron reuniones con los representantes de las familias y de las comunidades donde se les brindó una explicación detallada del estudio a realizar. Los padres o tutores y los niños fueron citados para el día del estudio en las escuelas o en las viviendas de los líderes de las comunidades visitadas. Previo consentimiento informado de los padres o tutores, se realizó una encuesta con preguntas cerradas por grupo familiar a fin de recabar información referente a las características socio-ambientales y sociosanitarias: edad, sexo, lugar de residencia, origen del abastecimiento de agua (naciente/arroyo, pozo comunal, pozo privado, agua corriente), tipo de piso de la vivienda (tierra, madera, cemento, ladrillo/cerámica/baldosa) y modalidad de eliminación de excretas (a ras del suelo, letrina comunal, letrina privada, fosa séptica).

\section{Análisis de las muestras}

Se analizaron las muestras de heces correspondientes a una sola deposición, debido a la dificultad de obtener muestras seriadas en la población estudiada. Para la recolección de las mismas fueron entregados envases apropiados con formol al 10\%. Para el análisis coproparasitológico de las muestras de heces se utilizó el método directo en solución salina fisiológica y coloración con lugol, el método de flotación de Willis y el de concentración éter formalina al $10 \%{ }^{25}$.

\section{Análisis estadístico}

Los datos fueron procesados mediante Epi-Info 3.5.3. Las asociaciones fueron analizadas por el test de $\chi^{2}$ y la prueba de Fisher según correspondía. Los resultados se consideraron significativos a un intervalo de confianza de $95 \%(\mathrm{p}<0,05)$.

\section{Asuntos éticos}

Los padres o tutores que aceptaron que los niños participaran del estudio firmaron un consentimiento informado. Los resultados fueron entregados a los padres o tutores y los niños fueron asistidos por el equipo médico

\begin{tabular}{|c|c|c|}
\hline \multirow[t]{2}{*}{ Distritos } & \multicolumn{2}{|c|}{ Comunidades } \\
\hline & Indígenas & No Indígenas \\
\hline Abaí & $\begin{array}{l}\text { Ypetimi } \\
\tilde{N} u \text { Apuá } \\
\text { Tajay }\end{array}$ & $\begin{array}{l}\text { Kilómetro } 9 \\
\text { Michimi } \\
\text { San Francisco } \\
\text { San Marcos } \\
\text { San Miguel } \\
\text { San Miguel La Esperanza } \\
\text { Villa Pastoreo }\end{array}$ \\
\hline Tavaí & Tuna'í & $\begin{array}{l}\text { San Francisco } \\
\text { Yvyhatí } \\
\text { Santa Ana }\end{array}$ \\
\hline
\end{tabular}

del Centro de Salud local, y se les proveyó del antiparasitario albendazol, $400 \mathrm{mg}$ diarios por vía oral durante 5 días y metronidazol $20 \mathrm{mg} / \mathrm{kg} /$ día durante 7 días. $\mathrm{El}$ protocolo fue aprobado por el Comité Ético del Instituto de Investigaciones en Ciencias de la Salud (IICS), con número de código $\mathrm{P} 32 / 2011$.

\section{Resultados}

La población total estudiada estuvo compuesta por 247 niños bajo 5 años de edad, indígenas y no indígenas; de los cuales 123 correspondían a niños indígenas y 124 niños a la población no indígena. Entre los niños indígenas, $43,1 \%$ (n: 53 ) pertenecía al sexo femenino y $56,9 \%$ (n: 70) al masculino, mientras que los niños no indígenas $40,3 \%$ (n: 50 ) correspondía al sexo femenino y $59,7 \%$ (n: 74) al sexo masculino. No se encontraron diferencias significativas entre los sexos respecto a las parasitosis. Con respecto a la edad de los niños estudiados, la media de los indígenas fue de $28 \pm 16,3$ meses y de los no indígenas $29 \pm 15,7$ meses.

\section{Características socio-demográficas}

En las comunidades indígenas, la mitad de los niños que bebían agua del Ycuá o arroyo se encontraban parasitados, así como la mitad de los que evacuaban a cielo abierto; las comunidades indígenas carecen de agua potable. Las comunidades no indígenas presentaron un mayor acceso al suministro de agua corriente; en estas comunidades encontramos viviendas con piso de ladrillo, cerámica o baldosa, y presencia de fosa séptica. Diferencias significativas con respecto a la parasitosis entre ambas poblaciones $(\mathrm{p}<0,05)$, se observaron en las comunidades de niños indígenas, que contaban con pozos y letrinas comunales respecto a las letrinas privadas de las comunidades de niños no indígenas, y en los niños 
Tabla 2. Parasitosis en niños indígenas y no indígenas según factores socio-ambientales y sanitarios

Factores socio-ambientales
y sanitarios

\begin{tabular}{cc}
\multicolumn{2}{c}{ Indígenas } \\
$\mathrm{n}$ total & Niños \\
de niños & parasitados
\end{tabular}

$$
\text { No indígenas }
$$

de niños parasitados

n total Niños

de niños parasitados

$$
\text { n (\%) }
$$

n (\%)

\section{Abastecimiento de agua}

\section{Naciente/arroyo}

Pozo comunal

Pozo privado

Agua corriente

Total

Tipo de piso de la vivienda

Tierra

Madera

Cemento

Ladrillo, cerámica, baldosa

Total

\section{Excretas}

A ras del suelo

Letrina comunal

Letrina privada

Fosa séptica

Total

\begin{tabular}{|c|c|c|c|c|c|}
\hline 55 & $35 \quad(51,5)$ & 3 & 2 & $(4,5)$ & ${ }^{*} \mathrm{NS}$ \\
\hline 62 & $30 \quad(44,1)$ & - & & - & 0,03 \\
\hline - & - & 85 & 28 & $(62,2)$ & \\
\hline 6 & $3 \quad(4,4)$ & 36 & 15 & $(33,3)$ & *NS \\
\hline 123 & $68(100)$ & 124 & & (100) & \\
\hline 91 & $53 \quad(76,8)$ & 61 & 19 & $(43,2)$ & 0,0005 \\
\hline 18 & $8 \quad(11,6)$ & 15 & 8 & $(18,2)$ & NS \\
\hline 14 & $8 \quad(11,6)$ & 31 & 14 & $(31,8)$ & NS \\
\hline & - & 17 & 3 & $(6,8)$ & \\
\hline 123 & $69(100)$ & 124 & & (100) & \\
\hline
\end{tabular}

*NS: No significativo.

\section{$9 \quad 5 \quad(7,2)$}

$114 \quad 64 \quad(92,8)$

- 116

$43(97,7)$

$8 \quad 1 \quad(2,3)$

$123 \quad 69(100) \quad 124 \quad 44(100)$
0,0006
Figura 2. Frecuencia de parasitosis en los niños indígenas ( $\mathrm{n}: 123)$ y no indígenas (n: 124), (p: 0,0006).

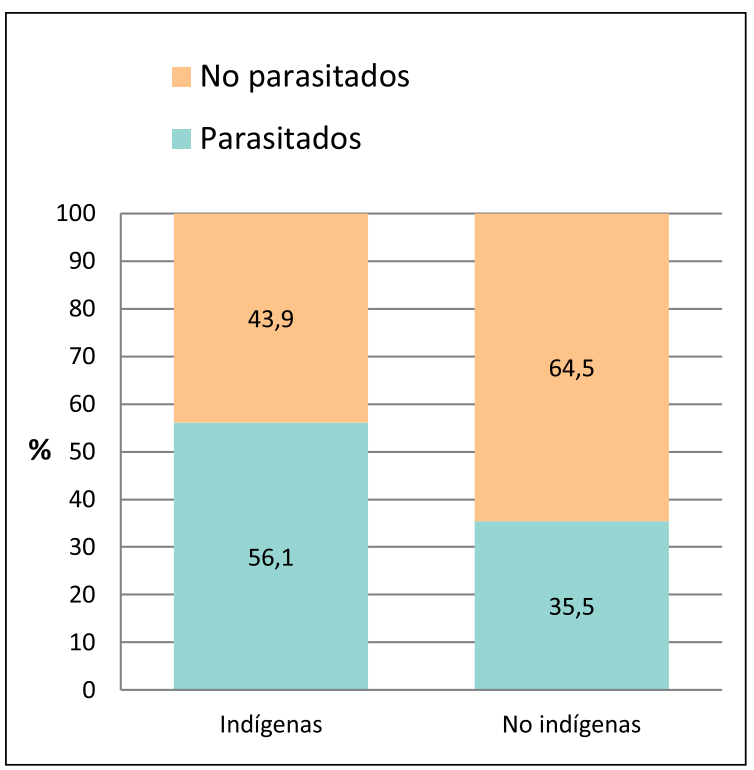

indígenas que habitaban viviendas con pisos de tierra. Para las demás variables estudiadas no se observaron diferencias significativas entre los niños indígenas y no indígenas en relación a la parasitosis (Tabla 2).

En la población de niños indígenas observamos una frecuencia de parasitosis de 56,1\% (69/123); y en los niños no indígenas una frecuencia de 35,5\% (44/124). Los niños indígenas y no indígenas mayores de 24 meses se encontraban significativamente más parasitados (Figura 2 y Tabla 3 ).

El monoparasitismo prevaleció en la población de niños indígenas y no indígenas. Hallamos una diferencia estadísticamente significativa en los niños indígenas poliparasitados con respecto a los no indígenas $(\mathrm{p}<0,05)$. En ambas poblaciones se encontró un predominio de protozoarios sobre helmintos (Tabla 4).

En los niños indígenas hallamos ocho especies de enteroparásitos mientras que en la no indígena fueron cuatro especies. Los parásitos más frecuentemente encontrados fueron los protozoarios Giardia lamblia y Blastocystis hominis en ambas poblaciones. En cuanto a los helmintos se encontró en los niños indígenas una predominancia de Hymenolepis nana, y en los niños no indígenas el helminto hallado fue Ascaris lumbricoides y sólo se registraron cuatro casos de poliparasitismo (Tabla 5).

Tabla 3. Presencia de parasitosis según edad en niños indígenas y no indígenas

\begin{tabular}{|lcc|}
\hline Grupo etario & $\begin{array}{c}\text { Indígenas } \\
\mathbf{n}(\%)\end{array}$ & $\begin{array}{c}\text { No indígenas } \\
\mathbf{n}(\%)\end{array}$ \\
$<$ a 24 meses & $17(24,6)$ & $18(40,9)$ \\
$\geq$ a 24 meses & $52(75,4)$ & $26(59,1)$ \\
Valor $p$ & $<0,001$ & $<0,03$ \\
Total & $69(100)$ & $44(100)$ \\
\hline
\end{tabular}

Tabla 4. Distribución del tipo de parasitosis en niños indígenas y no indígenas

\begin{tabular}{|lrrrrc|} 
Presencia de parásitos & \multicolumn{2}{c}{ Niños } & \multicolumn{2}{c|}{ Niños } \\
& \multicolumn{2}{c}{ indígenas } & \multicolumn{2}{c}{ no indígenas } \\
& n & $\%$ & n & $\%$ \\
Monoparasitismo & 42 & 60,9 & 40 & 91,0 \\
Poliparasitismo & 27 & 39,1 & 4 & 9,0 \\
Tipo de parásitos & & & & \\
Protozoarios & 47 & 68,1 & 39 & 88,6 \\
Helmintos & 8 & 11,6 & 5 & 11,4 \\
Protozoarios + helmintos & 14 & 20,3 & 0 & - \\
Total & 69 & 100 & 44 & 100 \\
\hline
\end{tabular}




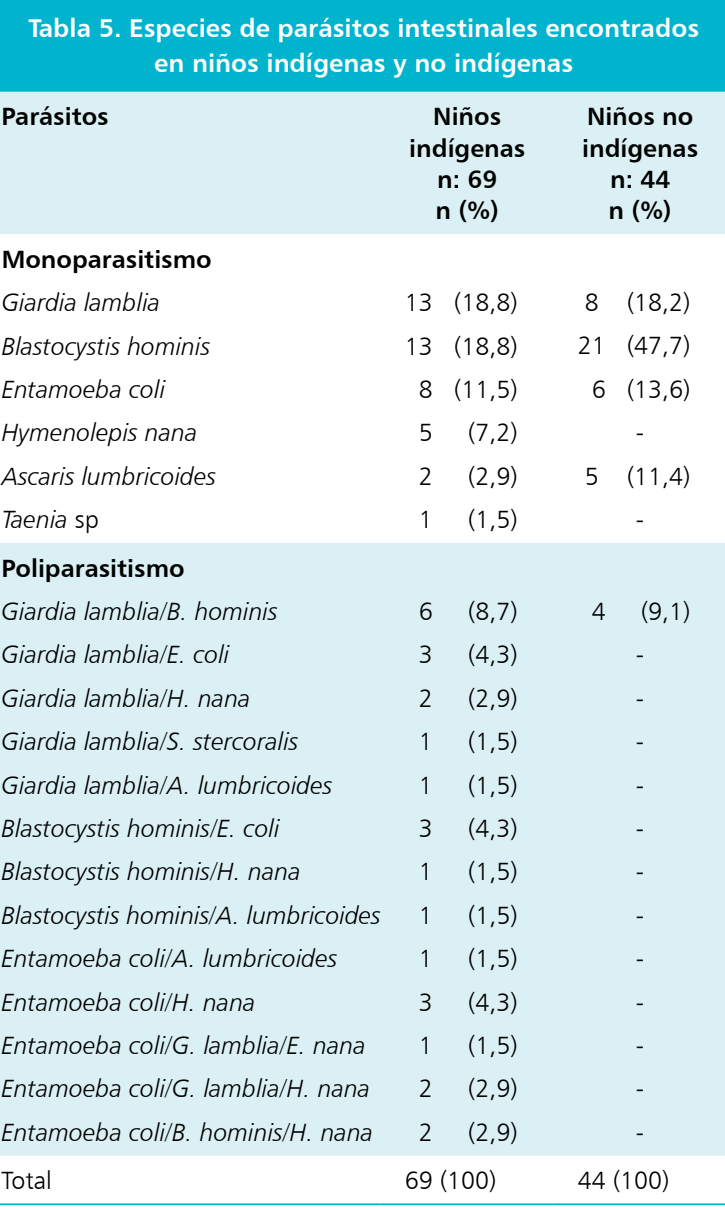

\section{Discusión}

Existen escasos estudios nacionales publicados, sobre parasitosis en niños bajo cinco años, de zonas rurales, así como datos actualizados de la situación. No se encontraron publicaciones sobre estudios parasitológicos en niños indígenas de este grupo etario en el Paraguay.

En cuanto a las condiciones de vida de los niños de las comunidades estudiadas, cabe resaltar las precarias condiciones en las que viven las comunidades indígenas, donde los niños presentaron asociaciones significativas entre parasitosis y las viviendas con piso de tierra, pozos y letrinas comunales; en principio se considera que cualquier vivienda con piso de tierra presenta una carencia ${ }^{26}$, así como la falta de acceso a agua potable y escasos hábitos higiénicos como lavarse las manos después de ir al baño, antes de tocar los alimentos o después de jugar con la tierra, los cuales constituirían factores que permitirían la transmisión de la infección. Aunque en este estudio la falta de hábitos higiénicos no fue una variable estudiada, cabe destacar que la mitad de los niños indígenas consumen agua de las nacientes o arroyos, las cuales quedan alejados de sus viviendas y debían transportar el agua en recipientes no siempre higienizados.

Un estudio realizado en México indica que las necesidades de la población indígena duplica a la población no indígena en relación a pisos de la vivienda, agua y drenaje ${ }^{26}$.

Estudios en otras comunidades indígenas presentaron porcentajes similares al nuestro referentes a las viviendas con pisos de tierra así como al abastecimiento del agua ${ }^{27}$. En cuanto a la eliminación de excretas los indígenas emberáchamí, presentaron también como el presente trabajo, asociación con las parasitosis, aunque sólo 4\% lo realizaban en pozos u otros medios ${ }^{28}$. En las comunidades indígenas de Ixhuapan y Ocozotopec la falta de drenaje alcanzaba entre 88 y $90 \%$, la mayoría de la personas iban al monte, mientras que en nuestra población la letrina comunal fue el medio de eliminación de excretas con mayor proporción ${ }^{27}$; estas variaciones podrían estar relacionadas a la situación geográfica, las costumbres y cultura de las etnias estudiadas.

Las comunidades no indígenas presentaron mejores condiciones de vida, que podría deberse a una mayor cercanía a las rutas y a los centros urbanos. Las viviendas poseían mayoritariamente pozo privado o agua corriente, letrinas privadas con infraestructura adecuada y fueron los únicos que contaban con pisos de ladrillo, cerámica o baldosa y fosa séptica en sus viviendas, lo que podría explicar el menor porcentaje de parasitosis en los niños no indígenas. Estudios realizados por Ávila y cols. ${ }^{29} \mathrm{y}$ Canese y cols. ${ }^{17}$, respecto a las condiciones de la vivienda, reportan frecuencias similares a la encontrada en nuestro trabajo, con respecto al acceso a agua potable y al piso de material utilizado, respectivamente.

Observamos en el presente estudio, una mayor frecuencia de parasitosis en los niños indígenas estudiados en comparación con los no indígenas ( $\mathrm{p}$ : 0,0006), similar al encontrado por Navone y cols. ${ }^{30}$, en la comunidad aborigen de Takuapí con respecto a la no aborigen, relacionadas posiblemente a la presencia de las mismas pobres condiciones de calidad de vida encontradas en nuestra población estudiada. Los datos provenientes de América Central y el resto de América Latina indican con claridad que el estado nutricional y de salud, así como las condiciones socio-económicas generales, son peores entre los indígenas que entre los grupos no indígenas ${ }^{4}$. Los niños indígenas estudiados viven en comunidades aisladas y alejadas, con caminos de difícil acceso en medio del monte, carecen de agua potable y servicios sanitarios adecuados.

Las frecuencias encontradas en los niños indígenas y no indígenas bajo cinco años del presente trabajo, 56,1 y $35,5 \%$, respectivamente, fueron superiores al porcentaje 
de morbilidad de parasitosis intestinal reportado a nivel nacional $^{5}$, y al porcentaje informado por la Organización Panamericana de la Salud (OPS) para América Latina ${ }^{4}$.

Aún siendo elevado el porcentaje de parasitosis encontrado en los niños indígenas estudiados en el Departamento de Caazapá, Paraguay, otros trabajos en países sudamericanos, en comunidades indígenas, reportan prevalencias de parasitosis superiores, con frecuencias que van desde 67 a $87 \%{ }^{31-33}$, en niños indígenas Warao y de la comunidad Yukpa, en edades comprendidas entre 0 y 15 años. Otros estudios realizados en comunidades indígenas de la etnia Emberá-chamí, en indígenas Mbyá Guaraní y en la etnia Emberá Katio y Kogui ${ }^{9,28,30}$ de todas las edades, presentaron frecuencias superiores a $80 \%$; estas diferencias podrían deberse a un mayor rango de edad incluidos en estos estudios, a las características geográficas y climatológicas de las regiones, al número de individuos que formaron parte del estudio y las diferencias culturales entre las etnias. Los pueblos indígenas tienden a vivir en zonas rurales a menudo aisladas, que los separa de los servicios de salud, con recursos económicos insuficientes y la falta de servicios de calidad y culturalmente apropiados. La mortalidad y la morbilidad de los lactantes y los niños constituyen todavía un problema grave para los pueblos indígenas ${ }^{4}$.

En los niños no indígenas de las comunidades rurales estudiadas, encontramos una frecuencia de parasitosis inferior al reportado en trabajos nacionales, como el de Pistilli y cols. ${ }^{15}$, realizada en niños de 6 a 10 años, de zonas aledañas al río Paraguay en la capital del país, al de Ramírez y cols. ${ }^{16}$, realizado en niños de 1 a 12 años procedentes del área urbana y al de Canese y cols. ${ }^{17}$, en niños de 4 a 15 años en población urbana y rural, de datos recopilados en la Cátedra de Microbiología de la Facultad de Ciencias Médicas de Asunción, Paraguay, entre los años 1995 y 1998. Estas diferencias encontradas en los estudios nacionales podrían deberse a la procedencia urbana o rural de los niños, a las zonas geográficas a las que pertenecen, al rango de edad estudiado, ya que en su mayoría en los trabajos nacionales mencionados fueron niños escolares, así como a la procedencia de los datos encontrados. Los niños de las comunidades estudiadas en este trabajo, por su corta edad, aún se encontraban bajo el techo familiar por lo que el trabajo se realizó in situ.

Frecuencias similares a nuestra población de niños no indígenas, fueron encontradas por Ávila-Rodríguez y cols. ${ }^{29}$, en niños no indígenas bajo cinco años de edad, de cuatro asentamientos en una zona urbana, y por Sánchez M. y cols. ${ }^{34}$, en edades comprendidas entre 3 y 19 años de comunidades rurales del altiplano de México. Sin embargo, en otros estudios latinoamericanos ${ }^{35-38}$ se observa un incremento en la frecuencia; y en la región, se encuentra una tendencia entre 60 y $74 \%$ de parasito$\operatorname{sis}^{30,40,41}$. La mayor variedad de especies encontradas en otras investigaciones, como es Enterobius vermicularis, el que fue un parásito frecuente en varias de ellas ${ }^{31,35-37}$ y la recolección de heces de más de una deposición, podrían haber influido en estas diferencias, ya que en nuestro estudio no se aplicó el test de Graham y la recolección de las muestras fue de una sola deposición, debido a las dificultades culturales por las edades tempranas de nuestros niños, y de accesibilidad a las zonas rurales para la recolección de las muestras. Cabe señalar que a causa de los propios ciclos biológicos de los enteroparásitos, existe una eliminación intermitente de los mismos, que favorece períodos negativos en los exámenes de heces, motivo por el cual la recolección de múltiples muestras aumenta la probabilidad del hallazgo; esta es una razón probable de la disminución del porcentaje de positividad de los exámenes en el presente estudio.

La menor proporción de infección parasitaria encontrada en los niños bajo 24 meses de edad, en ambas poblaciones, coincide con los hallazgos de otros investigadores, donde los menos afectados son los niños en edades más tempranas ${ }^{29,31-33,39,41}$. Otros autores reportaron una menor proporción de parasitosis en los niños preescolares en relación a los escolares ${ }^{34,38}$. Esto posiblemente debido a los cuidados maternos que reciben los niños en esta franja etaria, mientras que los de edades superiores presentan una mayor exposición a los factores que intervienen en la infección parasitaria a medida que crecen y se desenvuelven por ellos mismos ${ }^{1}$

No se observaron en ambas poblaciones de niños diferencias significativas respecto a la parasitosis con relación al sexo, en forma similar a otros estudios ${ }^{28,30,35,40,42}$.

En ambas poblaciones de niños indígenas y no indígenas encontramos un predominio de monoparasitismo, similar a otros trabajos realizados ${ }^{16,17,28,29,33,36}$. Con respecto al poliparasitismo observamos trece asociaciones de parásitos en los niños indígenas y una en los no indígenas, siendo la asociación más frecuente en ambas poblaciones la de G. lamblia y B. hominis, similar al encontrado por Pistilli y cols. ${ }^{15}$, en niños de zonas aledañas al río Paraguay en Asunción, Brito Núñez y cols. ${ }^{33}$, en indígenas Warao de Venezuela, y Vera $^{36}$ en niños de Lima, Perú.

Tanto en los niños indígenas como en los no indígenas se observó una mayor frecuencia de protozoarios sobre helmintos, similar al hallazgo de otros estudios en la región $^{9,28,32,33}$.

En los niños indígenas, los protozoarios predominantes fueron G. lamblia seguida del B. hominis. En países tales como Venezuela, Colombia, Bolivia y Argentina $a^{9,28,30-33,43}$ $B$. hominis fue el protozoario predominante mientras $G$. lamblia fue el tercero o cuarto prevalente. En los niños no indígenas, los protozoarios predominantes también fueron $B$. hominis y $G$. lamblia, acorde con los trabajos nacionales ${ }^{15-17}$ realizados entre los años 1997 y 1999 y similares a estudios realizados en los países latinoame- 
ricanos $^{35-41}$. Nuestros hallazgos revelan que continúan siendo los mismos agentes infecciosos los que a través de los años persisten en nuestro país, afectando la salud a estas poblaciones vulnerables. La presencia de estos protozoarios podría deberse a las aguas no tratadas que consumen o lavan sus alimentos, en las comunidades visitadas, que constituiría uno de los principales mecanismos de transmisión de estos parásitos, favoreciendo la transmisión de los mismos. Los protozoos generalmente se encuentran asociados a enfermedades transmitidas por el agua. En general en los países en desarrollo existe una mayor prevalencia de $B$. hominis que en los países desarrollados relacionado con la falta de higiene, la exposición a animales y el consumo de alimentos o agua contaminados $^{11,31,40}$.

Con respecto a los helmintos, los niños no indígenas presentaron una predominancia de $H$. nana seguida de A. lumbricoides, (llamado sevo'i pyta en nuestra lengua guaraní), parásitos predominantes en los trabajos de Brito Núñez y cols. ${ }^{33}$, Díaz I. y cols. ${ }^{31}$, y Luna Monroy y cols. $^{43}$; mientras que en los niños no indígenas el único helminto encontrado fue $A$. lumbricoides, similar al hallazgo, a nivel nacional, de Ramírez y cols. ${ }^{16}$, mientras que en el de Canese y Pistilli ${ }^{15,17}$ fue el segundo y tercero más frecuente respectivamente. Así mismo, se encontró entre los parásitos predominantes en países Latinoamericanos tales como Costa Rica, Argentina, Colombia y Venezuela ${ }^{30,31,33,44}$. Ascaris lumbricoides se contrae por ingestión de los huevos procedentes del suelo contaminado $^{3}$ mientras que $H$. nana es la infección por cestodos más frecuente en el que intervienen roedores e insectos que van a contaminar las aguas con quistes o embriones, el hombre puede ingerir los huevos bajo escasas condiciones de limpieza, como ser verduras crudas, agua contaminada o manos sucias, siendo liberadas las oncosferas en el intestino delgado ${ }^{45,46}$. Los niños son más susceptibles que los adultos en contraer la infección ${ }^{3}$. Según Saboya y cols., la prevalencia de infección por helmintos en América Latina y el Caribe se encuentra por encima de $20 \%{ }^{47}$, frecuencia similar a la observada en los niños indígenas del presente estudio.

Factores socio-sanitarios, ambientales y conductuales de las comunidades de niños indígenas y no indígenas estudiadas, como el suministro de agua no tratada, la deficiente infraestructura de las viviendas, las costumbres y la precaria eliminación de excretas, aumentan el riesgo de transmisión de las especies parasitarias halladas.

En el año 2013 el Ministerio de Salud Pública y Bienestar Social firmó un acuerdo con el Ministerio de Educación y Cultura, para llevar adelante el Programa de Desparasitación Escolar Masiva, dirigida a niños de entre 6 y 12 años, con la provisión del fármaco antiparasitario ${ }^{48}$.

Las enteroparasitosis siguen constituyendo un grave y persistente problema de salud en los países latinoa- mericanos como el nuestro, en especial en los pueblos indígenas que comparten características y pobres condiciones de vida. El hallazgo de una elevada frecuencia de parasitosis en la población infantil indígena, a expensas de los protozoarios, sería debida posiblemente a las malas condiciones socio-económicas y de saneamiento, la falta de suministro de agua potable, y favorecida por el difícil acceso de sus comunidades a los centros de salud, sumado a las limitantes lingüísticas o culturales.

El trabajo demuestra que los niños siguen portando las mismas especies parasitarias que las encontradas por estudios nacionales de años anteriores, y es un llamado de atención para redoblar esfuerzos en busca de reducir la infestación parasitaria.

Siendo la niñez un grupo vulnerable a las infecciones parasitarias, el presente trabajo provee información actualizada a las autoridades sanitarias sobre la problemática de la parasitosis en los niños bajo cinco años de edad, que contribuya en la formulación de políticas públicas que mejoren las condiciones socio-sanitarias de las comunidades rurales, favorezcan el acceso a agua potable, viviendas adecuadas, comunicación vial, adquisición de conocimientos sobre la transmisión parasitaria y promuevan hábitos saludables que beneficien el desarrollo de los niños mediante la prevención y el control de la parasitosis.

Agradecimientos. Los autores expresan su agradecimiento a las Docentes Investigadoras del IICS: María Mercedes Carpinelli, Elizabeth Orué, Nathalie Lampert, Mónica Sequera y Mónica Ruotti, a las Técnicas de Laboratorio: Stella Vázquez, Lorenza Servín, Blasia Cabral, Diana Ortiz y Ramona Castillo. A la enfermera Francisca Cantero. A la Fundación Acción contra el Hambre $(\mathrm{ACH})$ por la donación de medicamentos antiparasitarios y por el apoyo logístico.

\section{Resumen}

Introducción: Cifras oficiales de mortalidad en niños bajo 5 años de edad, en las Américas, refieren que la mayor parte de las defunciones fueron causadas por enfermedades infecciosas incluyendo las parasitarias. Objetivo: Evaluar la frecuencia de enteroparasitosis en población infantil vulnerable, indígenas y no indígenas, y sus características socio-ambientales. Pacientes $y$ Métodos: Se evaluaron 247 niños bajo 5 años de edad, de ambos sexos. Estudio descriptivo con componente analítico, de corte transverso. Se realizaron exámenes copro-parasitológicos y se aplicaron encuestas semiestructuradas para recoger datos socio-demográficos. Resultados: la frecuencia de enteroparasitosis en los niños indígenas fue de $56,1 \%$ y en los niños no indígenas de $35,5 \%$. En ambas poblaciones los patógenos más frecuentes fueron Gardia lamblia y Blastocystis hominis. 
Conclusión: Encontramos una elevada frecuencia de parasitosis en la población infantil indígena, a expensas de los protozoarios. Los niños no indígenas siguen portando las mismas especies parasitarias encontradas en estudios anteriores, sugiriendo la necesidad de implementar un mayor control y prevención. Existen escasos estudios en nuestro país sobre parasitosis en edades tempranas y no se cuentan con datos en la niñez indígena. Las pobres condiciones en las que viven favorecen el desarrollo de estas enfermedades.

\section{Referencias bibliográficas}

1.- Mehraj V, Hatcher J, Akhtar S, Rafique G, Beg M A. Prevalence and factors associated with intestinal parasitic infection among children in an urban slum of Karachi. Plosone 2008; 3 (11): e3680.

2.- Del Real S I, Sánchez Jaeger A, Barón M A, Díaz N, Solano L, Velásquez E, et al. Estado nutricional en niños preescolares que asisten a un jardín de infancia público en Valencia, Venezuela. Arch Latinoam Nutr 2007; 3 (57)

3.- Un Llamado a la Acción: Hacer frente a los helmintos transmitidos por el contacto con el suelo en Latino América y el Caribe. Pan American Health Organization. World Health Organization; 2011.

4.- La Salud en las Américas. Organización Panamericana de la Salud. Organización Mundial de la Salud. Washington D.C.; 2002. Publicación Científica y Técnica No 587, (1).

5.- Perfil de la salud ambiental infantil en Paraguay. Organización Panamericana de la Salud, Organización Mundial de la Salud, 2003. www. bvsde.ops-oms.org/bvsana/e/fulltext/perfiles/ paraguay.pdf

6.- Savioli L, Smith H, Thompson A. Giardia and Cryptosporidium join the 'Neglected Diseases Initiative'. Trends Parasitol 2006; 22 (5): 203-8.

7.- Barón M A, Solano L, Páez M C, Pabon M. Estado nutricional de hierro y parasitosis intestinal en niños de Valencia, Estado Carabobo, Venezuela An Venez Nutr 2007; 20 (1): 5-11.

8.- Urquiza Y, Domínguez M, Artiles M. Caracterización clínico-epidemiológica del parasitismo intestinal en niños de 0 a 5 años. Rev Cubana Med Gen Integr 2011; 27 (1): 105-13.

9.- Puerta L, Salazar L, Velásquez L, Vélez I. Estado actual de las parasitosis intestinales en cuatro comunidades indígenas de Colombia. Biomédica 2011; 31 (Supl. 3): 313-5.

10.- Perfil de la salud ambiental infantil en Paraguay. Organización Panamericana de la Salud, Organización Mundial de la Salud, 2003. www. bvsde.ops-oms.org/bvsana/e/fulltext/perfiles/ paraguay.pdf.

11.- Situación actual de la primera infancia y perspectivas de la atención integral en el Paraguay. Ministerio de Educación y Cultura. Ministerio de Salud Pública y Bienestar
Social. Secretaría Nacional de la Niñez y la Adolescencia. Fondo de las Naciones Unidas Para la Infancia (UNICEF, Paraguay), 2011.

12.- Agua potable y saneamiento en Paraguay. Servicio Nacional de Saneamiento ambiental (SENASA). Programa de Monitoreo Conjunto OMS/UNICEF (JMP/2006). www. senasa.gov.py/institucional/objetivos-generalesde-la-institucion.

13.- Política Nacional de Salud Indígena. Fondo de las Naciones Unidas para la Infancia (UNICEF). Ministerio de Salud Pública y Bienestar Social. Coordinación Nacional de Pastoral Indígena CEP (CONAPI). Paraguay para todos.

14.- Niñez indígena y escuela en Paraguay: un desafío pendiente. Fondo de las Naciones Unidas para la Infancia (UNICEF), 2008.

15.- Pistilli N, Malgarejo M, Ramírez A, Laviosa R, Sosa L. Parasitosis intestinal y anemia en escolares de zonas aledañas al río Paraguay en Asunción. Ann Rep 1997; 27-32.

16.- Ramírez A, Melgarejo M, Pistilli N, Díaz V, Sosa L, Martínez L, et al. Evaluación de métodos de diagnósticos de parásitos intestinales utilizados en el laboratorio de análisis clínicos. Ann Rep 1997; 39-43.

17.- Canese A, Barrios E, Castro L, Canese J. Prevalencia de parásitos intestinales en niños del Paraguay. Rev Parag Microbiol 1999; 1 (19): 14-8.

18.- Araujo P, Chamorro G, Torales J, Aguilar G, Weiler N, Britez E, et al. Prevalencia de parasitosis intestinal en niños escolares de la comunidad de Isla Saka. Rev Inst Med Trop 2011; Supp (6).

19.- Infecciones intestinales por protozoos y helmintos. Informe de un grupo científico de la OMS (Organización Mundial de la Salud); 1981. Ginebra. Serie de Informes Técnicos 666. Pp 7.

20.- El comunicador frente a la situación de la niña indígena. Herramientas para informar, educar y comunicar los derechos de la niñez y la adolescencia. Proyecto Comunicación y Movilización Social. Fondo de las Naciones Unidas para la Infancia (UNICEF). Guatemala, 2008. www.unicef.org.gt/1_recursos_unicefgua/ paquetesdecom/paquete_ninaindigena/pdf/ paq_situaciondela_nina_indigena_1.pdf

21.- Hulley S, Cummings S, Warren B, Grady D, Newman T. Diseño de investigaciones clínicas. $3^{\text {a }}$ ed. Philadelphia: Lippincott Williams \&Wilkins; 2007.
22.- Paraguay, Secretaría Técnica de Planificación de la Presidencia, Dirección General de Estadísticas, Encuestas y Censos. Censo Nacional de Población y Viviendas 2002. Resultados finales. Fernando de la Mora; 2004.

23.- Departamento de Caazapá (VI). Ubicación, geografía, accesibilidad, atractivos turísticos. Portal Guaraní. Dirección General de Estadísticas, Encuestas y Censos. Censo Nacional de Población y Viviendas 2002. Resultados finales. Fernando de la Mora; 2004.

24.- Atlas de las comunidades indígenas en el Paraguay. II Censo Nacional Indígena de Población y Vivienda. Dirección General de Estadísticas, Encuestas y Censos (DGEEC). Fernando de la Mora, Paraguay, 2004.

25.- World Health Organization. Basic laboratory methods in medical parasitology. Geneva: World Health Organization; 1991.

26.- Diagnóstico de las necesidades y rezago en materia de vivienda de la población en pobreza patrimonial. Secretaría de desarrollo social. Fideicomiso fondo nacional de habitaciones populares. México, 2009.

27.- Serrano A, Vázquez V, Montes M. La pobreza indígena en dos comunidades de Veracruz. Rev Cuicuilco 2006; 38 (13): 201-24.

28.- Cardona J, Marín D, Salazar E. Estudio ecológico sobre parasitismo intestinal, anemia y estado nutricional en indígenas emberá-chamí. Medicina y Laboratorio 2013; 5-6: 19.

29.- Ávila-Rodríguez A, Ávila-Rodríguez E H, Ávila-Pérez M, Araujo-Contreras J M, Rivas-Ávila E. Parasitosis intestinal y factores asociados, en niños menores de 5 años en cuatro asentamientos humanos irregulares de la ciudad de Durango, Méx. Enlaces académicos 2010; 1 (3): $15-25$.

30.- Navone G, Gamboa M, Oyhenart E, Orden A. Parasitosis intestinales en poblaciones Mbyá-Guaraní de la provincia de Misiones, Argentina: aspectos epidemiológicos y nutricionales. Cad. Saúde Pública 2006; 22 (5): 1089-100.

31.- Díaz I, Rivero Z, Bracho A, Castellanos M, Acurero E, Calchi M, et al. Prevalencia de enteroparásitos en niños de la etnia Yukpa de Toromo, Estado Zulia, Venezuela. Rev Med Chile 2006; 134: 72-8.

32.- Suárez-Díaz O, Atencio A, Carruyo M, Fernández P, Villalobos R, Rivero Z, et al. Parasitosis intestinales y tisulares y su relación con la eosinofilia en una comunidad indígena 
Yukpa de la Sierra de Perijá. Estado Zulia. Kasmera 2013; 1: 41.

33.- Brito-Núñez N, Arocha M. Prevalencia de parásitos intestinales en indígenas Warao de Cambalache, Estado Bolívar, Venezuela. Rev Biomed 2014; 25: 48-53.

34.- Sánchez de la Barquera M, Miramontes M. Parasitosis intestinales en 14 comunidades rurales del Altiplano de México. Rev Mex Patol Clin 2011; 1 (58): 16-25.

35.- Milano A M, Oscherov E B, Palladino A C, Bar A R. Enteroparasitosis infantil en un área urbana del nordeste argentino. Rev Medicina B Aires 2007; 3: 67.

36.- Vera D. Efectividad del tratamiento médico antiparasitario en niños de edad pre-escolar. Lima, Perú. Rev Peruana Epidemiol 2010; 1 (14): 72-8.

37.- Pérez M, Sánchez M, Cueto G, Mayor A, Fernández N, Alegret M. Intervención educativa y parasitismo intestinal en niños de la enseñanza primaria. Rev Cubana Med Gen Integr 2007; 23 (2).

38.- Solano L, Acuña I, Barón M, Morón A, Sánchez A. Influencia de las parasitosis intestinales y otros antecedentes infecciosos sobre el estado nutricional antropométrico de niños en situación de pobreza. Parasitol Latinoam 2008; 63: 12-9.

39.- Pezzani B, Minvielle M, Ciarmela M, Atezteguía M, Basualdo J. Participación comunitaria en el control de las parasitosis intestinales en una localidad rural de Argentina. Rev Panam Salud Publica/Pan Am J Public Health 2009; 26 (6): 471-7.

40.- Zonta M, Navone G, Oyhenart E. Parasitosis intestinales en niños de edad preescolar y escolar: situación actual en poblaciones urbanas, periurbanas y rurales en Brandsen, Buenos Aires, Argentina. Parasitol Latinoam 2007; 62: 54-60.

41.- Arévalo M, Cortés X, Barrantes K, Achí R. Prevalencia de parasitosis intestinal en niños de la comunidad de Los Cuadros, Goicoechea, Costa Rica. 2002-2003. Rev Costarric Cienc Méd 2007; 1-2 (28): 37-45.

42.- Giraldo J, Lora F, Henao L, Mejía S, Gómez J. Prevalencia de giardiasis y parásitos intestinales en preescolares de hogares atendidos en un programa estatal de Armenia, Colombia. Rev Salud Pública 2005; 7 (3): 327-38.

43.- Luna Monrroy S, Jiménez S, López R, Soto M,
Benefice E. Prevalencia de parasitismo intestinal en niños y mujeres de comunidades indígenas del río Beni. Rev Visión Científica 2007; 2 (1): 37-46.

44.- Carmona J, Uscátegui R, Correa A. Parasitosis intestinal en niños de zonas palúdicas de Antioquía. Rev Iatreia 2009; 1 (22): 27-46.

45.- Romero J, López M. Parasitosis intestinales. En: Serie protocolos de la Asociación Española de Pediatría. Protocolos diagnósticos-terapéuticos de Gastroenterología, Hepatología y Nutrición pediátrica SEGHNP-AEP. $2^{\mathrm{a}}$ edición. Madrid: Editorial Ergón; 2010.

46.- Blanco J, Galiano J. Atlas de coprología. Digestión y parásitos. $1^{\mathrm{a}} \mathrm{ed}$. Madrid: Editorial Garsi; 1989.

47.- Saboyá M I, Catalá L, Nicholls R S, Ault S K. Update on the mapping of prevalence and intensity of infection for soil-transmitted helminth infections in Latin America and the Caribbean: A call for action. PLoS Negl Trop Dis 2013; 7 (9).

48.- Ministerio de Salud Pública y Bienestar Social. Dirección General de Promoción de la Salud. Campaña Nacional de Desparasitación Escolar, 2014. 